\title{
Fiebre prolongada en preescolares y escolares
}

\author{
Drs. Fernando Moya V., $\left({ }^{* *}\right)^{*}$ Eduardo Talesnik G.," Rafael Larco C. *
}

\begin{abstract}
A retrospective study was performed in 62 cases of Protunged Fever (PF). 16 were 3 to 6 years old and 46 were 7 to 14 years old, $56.5 \%$ werc females.

Infectivus diseases cuused $77,4 \%$ of all cases of PF, with Typhoid Fever (64.5\%), pressumed Viral diseases $(10.4 \%)$ and Urinary Tract Infection $10.4 \%$ ) being the most common. There were 2 Collagen-Inflamatory diseases, one case of Drug Fever due to Phenobarbital and au Malignancies. 17.8\% of al] euses did not have y final diagnosis.

There was some relationship bet ween infectious diseases and season of the year. Signs and symptorns were helplul in certain cases, but contradictury in uthers. Alsu there was a tertain lack of specificily of laboratory findings.
\end{abstract}

La fiebre es un signo muy frecuente en la consulta pediátrica, que puede ser desencadenada por múltiples etiologías y que, en ocasiones, se prolonga por varios días sin una explicación clara, constituyendo motivo frecuente de hospitalización.

Diversas experiencias extranjeras en grupos pediátricos ${ }^{(1,2,3)}$ señalan a la TBC y a cuadros virales como las etiologías más frecuentes, relegando a lugares muy secundarios a la Fiebre Tifoidea, que en nuestro país probablemente ocupa un lugar más relevante.

Este hecho, sumado a las esporádicas publicaciones nacionales sobre el tema, motivaron nuestro trabajo.

\section{MATERIAL Y METODO}

Se revisaron las fichas de 62 niños hospitalizados en la Unidad de Segunda Infancia del Hospital Josefina Martínez de Ferrari, entre los meses de junio de 1977 a junio de 1978 y que cumplian con el criterio de tener $T^{\circ}$ Axilar mayor a $38^{\circ}$ (o su equivalente), por 7 días mínimo, independiente del diagnóstico final.

Los casos analizados fueron divididos en Preescolares (3-6 años) y Escolares (7-14 años), y además fueron agrupados por etiología en 5 grupos, de acuerdo a la biteratura pediátrica sobre el tema:

1. Enfermedades Infecciosas.

2. Enfermedades del Colágeno.

3. Neoplasias.

4. Misceláneas.

5. Causa desconocida.

Por tratarse de un estudio retrospectivo, no se contó con serología para virus y el diagnóstico final

"Servicio de Pediatría Hospiral Sótero del Róo, Departamento de Pediatría - Escueja de Medicina Universidad Católica de Chile.

*mBecado de Pediatria - Unirersidad Católica de Chile. de etiología viral se estableció por un cuadro clínico y hallazgos de laboratorio compatibles, además de la exclusión de otras patologías.

\section{RESULTADOS}

De los 62 casos estudiados hubo ur $56,5 \%$ de sexo femenino; 16 pacientes tenían entre 3 y 6 años, y la mayor parte (46 pacientes) tenian entre 7 y 14 años. (Tabla 1.)

TABLA I

FIEBRE PROLONGADA EN PRE-ESCOLARES Y ESCOLARES DISTRIEUCION POR SEXO Y EDAD : 62 CASUS :

\begin{tabular}{|c|c|c|c|}
\hline EDAD SEXO & 0 & 0 & TOTAL \\
\hline $3-6 . \mathrm{a}$ & 8 & 8 & 16 \\
\hline $714 \mathrm{a}$ & 19 & $2 ?$ & 66 \\
\hline TOTAL & 27 & 35 & 62 \\
\hline
\end{tabular}

Las causas infecciosas fueron las más frecuentes, con un total de 48 casos, $77 \%$ de los cuales tenian entre 7 y 14 años. La Fiebre Tifoidea fue responsable de 31 casos, con sólo 19,3\% entre 3 y 6 años. (Tabla 2.) Otras causas infecciosas de menor importancia fueron la Infección del Tracto Urinario (ITU) y "cuadros presumiblemente virales", entre otras. Además hubo un caso de Absceso Hepático Piógeno a Citrobacter en un escolar de 12 años con fiebre, palidez y compromiso del estado general de un mes de evolución, con masa abdominal al examen físico.

Sólo hubo 2 casos de Colagenopatías, ambos en el grupo de mayor edad, y correspondieron a una Artritis Reumatoidea Juvenil (ARJ), en una niña de 7 años con fiebre de más de un mes de duración, con 
artralgias y artritis de aparición tandía; y a una $\mathrm{En}$. fermedad Reumática (ER), en una niña de 13 años, también con manifestaciones articulares.

No hubo Neoplasias y finalmente quedaron 11 casos sin diagnóstico, que corresponden a un $17,8 \%$ del total.

TABLA II

FIEDFr PROLONGADA EW PRE.ESCOLARES Y ESCOLARES DISTRIEUCION POR CAUSA Y POR EDAD ( 62 CaSOSI

\begin{tabular}{|c|c|c|c|}
\hline CAUSAS EDAD & $3-6 \bar{a}$ & $7-19 \bar{a}$ & TOTAI. \\
\hline INFECCIONAS & il & 37 & 48 \\
\hline F. TIFOIDIFA & 6 & 25 & 31 \\
\hline I.T.U. & 2 & 3 & 5 \\
\hline VTROSIS & 1 & 4 & $s$ \\
\hline SEUIMONIA & - & 3 & 3 \\
\hline ESTREF'TOCOCJA & 2 & - & 2 \\
\hline$T B C$ & - & I & ] \\
\hline ABSCESO HEPAЛCO & - & I & 1 \\
\hline COLAGENOPATAS & . & 2 & 2 \\
\hline A. REUMATOIDE & - & 1 & 1 \\
\hline E REUMATTCA & - & 1 & 1 \\
\hline NEOPI ASIAS & - & - & - \\
\hline MSCEL ANEAS & 1 & - & 1 \\
\hline FIEFRE POR DROGAS & 1 & - & 1 \\
\hline DESCONOCIDAS & 4 & 7 & 11 \\
\hline TOTAL & 16 & 46 & 62 \\
\hline
\end{tabular}

\section{Duración de la fiebre}

En 39 casos, la fiebre tuvo una duración de 1 a 2 semanas y, entre ellos, el $79.5 \%$ correspondió a cuadros infecciosos, entre los cuales estuvo la totalidad de Cuadros Presumiblemente Virales y Estreptococias, y el $80 \%$ de las ITU.

Sólo hubo 2 casos con más de 4 semanas de fiebre, el Absceso Hepático Piógeno y la Artritis Reumatoidea Juvenil. (Tabla 3.)

\section{Distribución estacional}

Las 3/4 partes de los casos se agruparon en primavera y verano. Se encontraron algunas relaciones interesantes entre época del año y causas infecciosas. La Fiebre Tifoidea tue responsable de entre las 2/3 a 3/4 partes de los casos de fiebre prolongada en primavera y verano, en cambio sojo hubo un caso de
T A BLA III

figere prolongaua en fre-escolares y escolares CAUSAS INFECCIOSAS Y DURACION DE LA FIEGRE EN SEMANAS.

\begin{tabular}{|c|c|c|c|}
\hline CALISAS: & $1-2 s$ & $2 \cdot 4+s$ & $1<s$ \\
\hline F. TIFOMLA & 12 & $1\}$ & - \\
\hline I'C L: & a & 1 & - \\
\hline Vthosis: & 5 & - & - \\
\hline NLC:MLNIA & 2 & 1 & - \\
\hline I:SIREPTOCOCIA & 2 & - & - \\
\hline T.BC. & . & 1 & - \\
\hline ARSCliSO HEPATLCO & - & . & 1 \\
\hline TOTAI. & 31 & 16 & 1 \\
\hline
\end{tabular}

Fiebre Tifoidea de un total de 6 casos de fiebre prolongada en invierno.

El $72,7 \%$ de los cuadros febriles infecciosos que comprometían la vía aérea se concentraron en invierno y primavera. (Tablas 4 y 5 .)

\section{Sintomas y signos}

La totalidad de los cagos tuvo fiebre y más del $80 \%$ de ellos manifestaron Anorexia, Adinamia y Fatigabilidad.

Los sintomas relacionados al Aparato Digestivo fueron los más frecuentes $(75,6 \%)$, pero el único específico fue el hallazgo de masa abdominal que se pesquisó en el caso de Absceso Hepático Piógeno.

La tos fue el síntoma más frecuente del Aparato Respiratorio (19 casos), pero sólo en $1 / 3$ de los casos se relacionó a un diagnóstico final primariamente respiratorio.

$$
\text { TABLA IV }
$$

FIEGRE PROLONGADA EN PRE-ESCOLARES Y ESCOLARES DISTRIEUCION ESTACIONAL Y CAUSAS 62 CINOS

\begin{tabular}{|c|c|c|c|c|}
\hline CANA DSTACJN & |NYTERINNo) & DRIMAYT:RA & IITRANO & Goxis \\
\hline NHF-C CIONFS & 6 & 20 & 19 & 3 \\
\hline COI_AGFNOIPA A AS & . & - & I & 1 \\
\hline NTGPJ_ASTAS & - & - & - & - \\
\hline MisC IL1.ANGAS & 1 & $\cdot$ & - & - \\
\hline DESt:GNOC:IDAS & 3 & 3 & $i$ & 1 \\
\hline TOTAL. & 10 & 23 & 24 & 5 \\
\hline
\end{tabular}


TA B L A V

FIEERE PROLONGADA EN PRE-ESCOLARES Y ESCOLAFES DISTRIBUCION ESTACIONAL Y CAUSAS INFECCIOSAS (4Zे ca.s.

\begin{tabular}{|c|c|c|c|c|}
\hline CALES FSTACION & [NVTERNo) & PRIBAVERA & YERAND & o'tond \\
\hline F. $\operatorname{Tr}(n)+1$ & $l$ & i马 & 15 & 2 \\
\hline $1.1: 2$. & 2 & 1 & 2 & . \\
\hline V1R0S1S & 1 & 3 & 1 & - \\
\hline NELERONLA & . & 2 & . & 1 \\
\hline STRFTTCKKA & $i$ & 6 & - & - \\
\hline T.D.C. & * & - & l & - \\
\hline IHNCW HEPATISW & 1 & - & . & $\cdot$ \\
\hline
\end{tabular}

Siete pacientes presentaron sensibilidad en fosas lumbares y dolor intenso a la Puño Percusión Lumbar, sóbo 4 de ellos, que correspondian a 2 casos de ITU, 1 neumonía y 1 caso de etiología desconocida.

Hubo 5 pacientes con sintomatología articular, pero sólo los 2 que presentaban artritis correspondieron a colagenopatías, 1 ARJ y 1 E. Reumática.

Manifestaciones cutáneas presentaron 15 pacientes, correspondiendo el 93,3\% a casos de Fiebre Tifoidea (Roséolas en 12 casos y Púrpura en 2 casos). El cuadro restante correpondió a un Exantena Maculopapuloso por drogas (Luminal).

Adenopatías se pesquisaron en 4 pacientes, pero no tuvieron relación con el diagnóstico final.

\section{Laboratorio}

A todos los pacieztes se les practicó hernograma. De ellos, 33 casos tuvieron RGB en límites normales, 10 tuvieron máa de $10.000 \mathrm{~GB} / \mathrm{mm}^{3}$, que inclúan 8 causas infecciosas, ninguna Fiebre Tifoidea entre ellas, además de la ARJ y 2 de etiología desconocids.

Hubo 19 casos con menos de $5.000 \mathrm{~GB} / \mathrm{mm}^{3}$; entre estos últimos, 12 tenían además desviación a izquierda, correspondiendo en un 83,3\% a Fiebre Tifojdea.

La VHS se practicó en 53 casos. En 8 casos estuvo bajo $15 \mathrm{~mm}$./hr., 25 casos tuvjeron entre $16 \mathrm{y}$ $50 \mathrm{~mm}$. $/ \mathrm{hr}$., 17 cagos tuvieron entre 51 y $100 \mathrm{~mm}$. $/ \mathrm{hr}$. y sólo hubo 3 casos con VHS mayor de $100 \mathrm{~mm}$./hr., que incluían la $\mathrm{E}$. Reumática y 2 casos de etiología desconocida.

De 7 casos con VHS mayor de $50 \mathrm{~mm} . / \mathrm{hr}$. y más de $10.000 \mathrm{~GB} / \mathrm{mm}^{3}{ }^{3}$, 5 correspondieron a causas infecciosas y 2 no tuvieron causa conocida.

El Sedimento Lirinario estuvo alterado en $7 \mathrm{ca}$ sos, incluyendo 4 ITU, 1 Fiebre Tifoidea y 2 causa desconocida. Hubo 1 ITU con sedimento urinario normal repetido.

Estudio radiológico de tórax se practicó en $\mathbf{1 5}$ casos y fue $(+)$ en sólo 4, que correspondían a 1 TBC y 3 Neumonías.

Mielograma se hizo en 1 paciente, que tuvo un Púrpura Trombopénico por Fiebre Tifoidea. Además se realizó una Cintigrafía Hepática en el paciente con masa abdominal en relación al hígado y que resultó ser un Absceso Hepático Piógeno.

\section{DISCUSION}

Desde hace mucho tiempo la fiebre ha sido reconocida como manifestación de múltiples etiologías que actúan a través de producción de Pirógenos, Exógenos y Endógenos e interacción sobre el Centro Ter. morregulador del Hipotálamo Anterior. '(F') (Figura 1.)

Numerosas experiencias nacionales y extranjeras, tanto en niños como en adultos, han analizado los cuadros febriles prolongados y señalan a los cua dros infecciosos como la causa más frecuente de ellos, con un porcentaje variable de Colagenopatías y Neoplasias. ${ }^{\{2,3,4,5,6,7,8,20\}}$ Además, varios autores concuerdan en que habitualmente se trata de enfermedades comunes con manifestaciones atípicas ${ }^{(2,5,9)}$ y que se ven con mayor frecuencia en el sexo masculino. ${ }^{(2,4)}$

Nuestra revisión confirma a las infecciones como la causa más frecuente de fiebre prolongada, con $77,4 \%$ del total de casos, pero demuestra una menor frecuencia de Colagenopatías $(3,2 \%)$ e inexistencia de Neoplasias, hecho que se contrapone a otras publicaciones sobre el tema. (2. 3, 20) Además hubo ligero predominio del sexo femenino en la serie (56,5\%), que se acentuó en patologías como ITU, en que el $\mathbf{8 0 \%}$ de ellas se presentaron en niñas.

En nuestro pás ya ha sido señalada la Fiebre Tifoidea como la causa infecciosa más frecuente de fiebre prolongada, ${ }^{(7)}$ hecho que con mucho se corrobora en nuestra revisión (50\% del total de los casos) y que no se evidencia en publicaciones extranjeras, $^{(2,4,6,20)}$ seguramente por la menor incidencia de ella en los respectivos. ${ }^{(9,10,11)}$ A pesar de que las 4/5 partes de los casos de Fiebre Tifoidea se presentaron entre los 7 y 14 años, lo que es habitual, $\left.{ }^{(11,} 12\right)$ la frecuencia relativa con respecto a otras causas infecciosas fue hastante parecida en ambos grupos etarios ( $55 \%$ entre 3 y 6 años y $68 \%$ entre 7 y 14 años). 
FIE BRE

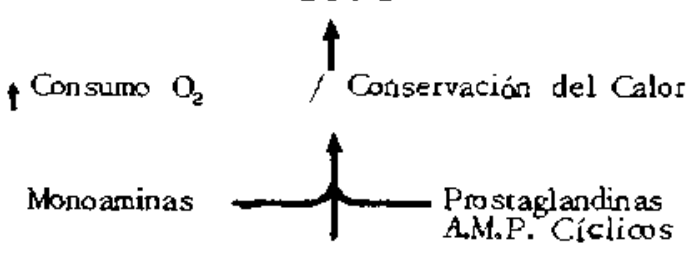

\section{CENTRO TERMO REGULADOR}

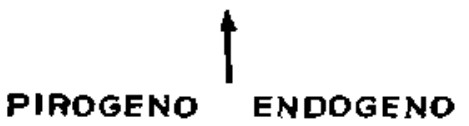

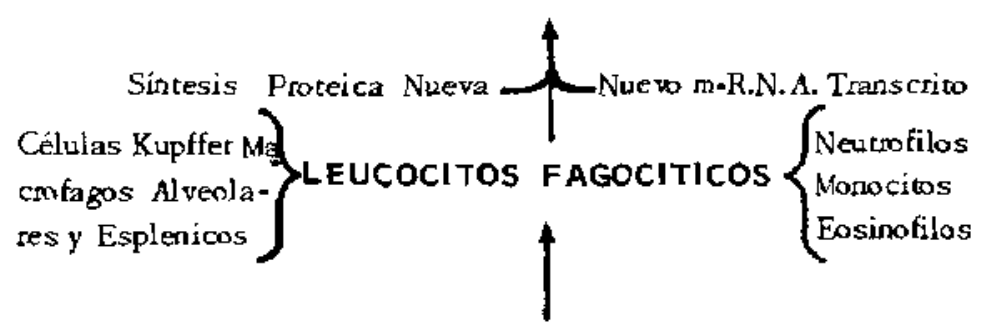

\section{PIROGENOS EXOGENOS}

Virus, Bacterias, Productos Bacterianos, Hongos

Endotoxinas, Etiocolanolona, Complejos $\mathrm{Ag}-\mathrm{Ac}$,

Antígenos (vía linfokinas de linfocitos sensibilizados).

Figura 1. Mecanismo propuesto paro la producción de Fiebre.

En lugares más secundarios se agrupan las ITU y cuadros infecciosos que comprometen la Vía Aérea (Virosis, Neumonías, Estreptococias y TBC) que han sido señalados como más frecuentes en la literatura. ${ }^{\{2.3}$, 20) Estos últimos cuadros se concentran en los meses de invierno y primavera, lo que puede constituir una orientación diagnóstica inicial. No tenemos una explicación clara para la existencia de sólo un caso de TBC, patología aún frecuente en nuestro medio.

Es necesario destacar la aparición de un caso de Absceso Hepático Piógeno a Citrobacter, patología que ya ha sido reconocida en la literatura extranjera como causa de fiebre prolongada, ${ }^{(13}$, 19) en nuestro caso, el hallazgo de Masa Abdominal, en relación al higado, Leucocitosis con VHS elevada y Cintigrafia Hepática sugerente fueron la clave del diagnóstico que se comprobó con Laparatomía Exploradora y Drenaje Quirúrgico y que correspondió al único caso sometido a cinugia en nuestra serie.

La incidencia de las Colagenopatías en la infancia no se conoce con exactitud en nuestro medio. En prácticamente todas las revisiones extranjeras de fiebre prolongada se presentan en porcentajes variables mayores que el nuestro, ${ }^{(2,3,6, ~ B . ~ 20) ~ y ~ p a r a ~} \mathrm{Mc}$ Clung, ${ }^{(6)}$ la ARJ constituye la causa más frecuente de fiebre prolongada. Aunque en nuestra casuística las colagenopatías aparecen con menor frecuencia $(3,2 \%)$, al igual que en las referencias citadas, afectan preferentemente a los pacientes de más de 7 años, y se deben considerar especialmente en aquellos casos en que se han descartado las causas infecciosas más frecuentes, que presentan, además, sigrnos claros de Artritis al examen físico. Nuestros dos casos correspondieron a una E. Reumâtica y una ARJ. En el primer caso, el diagnóstica se estableció en base a los criterios de diagnósticos aceptados universalmente. ${ }^{17)}$ La ARJ se puede presentar como un cuadro de fiebre prolongada, pudiendo tener episodios repetidos de fiebre y nush cutáneo, con Artritis mínima o con Artritis franca que aparece en un plazo de meses o años, desarrollando artritis crónica. En esta forma de presentación, las alteraciones de laboratorio características, Anticuerpos Antinucleares, Factor Reumatoideo, etc., son habitualmente negativas. ${ }^{(15.16)}$ 
Fiebre por drogas es un diagnóstico difícil y muchas veces de exclusión, y puede aparecer en relación a gran número de fármacos. ${ }^{14,}{ }^{19)}$ En nuestro caso, se trató de paciente de 3 años y medio, en tratamiento con Luminal, que presentó cuadro febril y exantema maculopapuloso generalizado, sin otros síntomas nj signos, con exámenes de Laboratorio dentro de límites normales, y en que cayó la fiebre espontáneamente 24 horas después de suspender el Luminal. Este barbitúrico puede causar cuadros febriles monosintomáticos de difícil diagnóstico, que obligan finalmente a su suspensión.!'18)

Los sintomas y signos pueden ser una excelente orientación diagnóstica en determinados casos, por ejemplo: hallazgo de Artritis, masa hepática, roséolas; pero también resultan no orientadores en otros, como los sintomas del Aparato Respiratorio, en que sólo $1 / 3$ de los casos que los presentaban se relacionaron a un diagnóstico final primariamente respiratorio.

Los exámenes de laboratorio corrientes también pueden tener resultados muy variables, ${ }^{(21}$ pero cuando hay alteraciones como Leucopenia con desviación a izquierda o asociación de Leucocitosis con VHS elevada, se puede lograr una adecuada presunción diagnóstica. Exámenes más específicos, como Mielogramas y Cintigrafia, fueron positivos, pero fueron solicitados con una orientación diagnóstica previa.

Finalmente, y al igual que todas las revisiones citadas, ${ }^{(2.3 .4,6,7,8,20)}$ un porcentaje variable de casos queda sin un diagnóstico etiológico, y es nuestra opinión que sólo un estudio prospectivo que incluya serología viral y un seguimiento prolongado puede hacer disminuir esta cifra de casos sin diagnóstico.

\section{RESUMEN}

Se presenta el análisis retrospectivo de 62 casos de fiebre prolongada, 16 entre 3 y 6 años y 46 entre 7 y 14 años, $56,5 \%$ de sexo femenino.

Las infecciones fueron responsables del $77,4 \%$ de los casos, siendo la Fiebre Tífoidea $(\mathbf{6 4 , 5 \%}$ ), los cuadros presumiblemente virales $(10,4 \%)$ y la infección urinaria $(10,4 \%)$ los cuadros infecciosos más frecuentes. Hubo 2 Colagenopatías y 1 fiebre por drogas (Fenobarbital). No hubo Neoplasias en la serie y un $17,8 \%$ de los casos quedaron sin diagnóstico.
Hubo cierta relación entre época del año y causas infecciosas. Los sintomas y signos resultaron de utilidad en determinados casos, pero fueron contradicturios en otros. En general hubo poca especificidad de los hallazgos de laboratorio.

\section{REFERENCIAS}

1 Dimarello, Ch., and Wolf, R. "Pathogenesis of Fever in man". New Engl. J. Med. 298 (1) $607-612$, March 16, 1978.

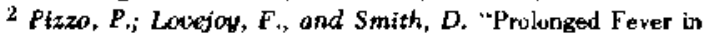
Children Review of 100 cases". Pediatrics 55 (4): 4686-473, April 1975.

3 Calderón, E., y cols. "Estudio prospectivo de pacientes con fiebre prolongada". Bol. Hosp. Inf. Mex. XXII 16): L00'-1019. Nov.-Dic. 1975

4 Serafin, $E_{\text {; }}$ Espinoza, E., y Gutierrez, G. "Síndrome febril de etiolugía por determinar". Bol. Hosp. Inf. Mex. XXIII (1): 79-90. enero-febrero 1976.

5 Cune, Th. "Children with Fever". Pediatries 43 (2): 290293. February 1969.

6 Mc Clung, /. "Prolonged Fever of Unknown origin Children". Ann. J. Dis, Child. 124: 544, February 1972.

7 Soto, S, y Vega, E. "Sindrorac febril prolongado". Rev. Méd. Chile 100: 37, 1972.

\& Petersdoof, $\boldsymbol{R}$, and Beeson, P. "Fever of Unexplained Oripin. Keport of 100 cases". Medjcine 40: 1, 1961.

9 Nelson. "Textbonk of Pediatrics". W' B. Saunders Company 1lth Edition: 704, 1979.

10 Krugman. "infections Diseases of Children". The C. V. Mosby Company 6th Editiun: 295, 1977.

11 Semipario Fiebre Tifoidea en Chile. Instituto Bacteriadógico de Chile - 1978.

12 Menaghello. "Pediatría". Editorial Intermédica 1." edición, 1972.

13 Vanni, L., et als, "Solitary Pyogenic Abacess in Children". Am. J. Dis. Child, 132: 1141-1142, Nav. 1978.

14 Kaplan, S. L; Feigin, L. D. "Pyogenic Liver Abscess in Normal Children with Fever of Unknown Origin". Pediatrics 58: 614-620, 1976.

15 Calobro. J. J. "Proceedings of the Conference on The Reumatic Disease of Childhood". Arthritis and Rheurnatism 20 (2): 237, Suppl. Mareh 1977.

16 Calobro, J. J., and Marchesano, J. M. "Fever aveociated with Juvenil Rheumaloid Arthritis". New Engl. J. Med. 276: 11-18, 1967.

17 Markoutitz and Cordis. "Rheunatic Fever", Major Problems in Clinical Pediatrics. W. B. Saunders Company. 1972.

18 Middleton, E., Reed, C, ond Ellis, E. "Allergy: Principles and Practice". The C. V. Mogby Company: 1144, 1978.

19 Kasik, J., and Thompson, J. "Allergic Reactions to Antibioties". Med. Clin. North. Am. 54 (1): 59. Januery 1970.

20 Cruz Guerrero, et al. "Fiebre prolongada de origen degconocido en el niño". Resumen Revisia de Revistas. Rev. Chil. Ped. 50 (3): 94, 1979.

$21 \mathrm{Wu}, E^{*}$, et al. "Sindrome febril prolongado en el preescolar y en el escalar". Bol. Hosp. San Juan de Dios, 26: 232-238, 1979.

22 Martinez, $F$., y Cabrera, $A$. "Estudio de 290 enfermos hospitslizados con el diagnóstico de 'Estado Infeccioro".' Rev, Chil Pediatr. 27: 397-403, 1956. 